\title{
O ModelCiências - um portal para o projeto Modelagem Semiquantitativa e Quantitativa na Educação em Ciências ${ }^{1}$
}

\section{The ModelCiências - a portal for the Semi Quantitative and Quantitative Computational Modeling in Science Education Project}

\author{
Arion de Castro Kurtz dos Santos* \\ André Prisco Vargas** \\ Odorico Machado Mendizabal ${ }^{* * *}$ \\ Carlos Alberto Barros Cruz Westhead Madsen ${ }^{* * * *}$
}

\begin{abstract}
RESUMO
$\mathrm{O}$ artigo apresenta alguns fundamentos da modelagem computacional, bem como algumas das ferramentas que estarão disponíveis no ModelCiências, que é um portal do projeto Modelagem Computacional Semiquantitativa e Quantitativa na Educação em Ciências do Plano Sul de Pesquisa e Pósgraduação do CNPq. O artigo apresenta, também, as características dos cursos a serem disponibilizados no portal, aspectos técnicos de sua construção e o atual estágio de desenvolvimento do projeto.

Palavras-chave: Modelagem, computador, ferramentas computacionais.
\end{abstract}

1 Trabalho financiado pelo CNPq.

* Ph.D. em Ciência da Educação pela University of London - Inglaterra. Professor Titular do Departamento de Física da Fundação Universidade Federal do Rio Grande Furg.dfsarion@furg.br

** Bolsista AT do CNPq, aluno de Engenharia da Computação da Furg. andrevar@ecomp.furg.br

*** Bolsista AT do CNPq, aluno de Engenharia da Computação da Furg. odo_mm@yahoo.com.br

****Bolsista Voluntário, aluno de Engenharia da Computação da Furg. carlos@ceamecim.furg.br 


\begin{abstract}
This paper presents some computational modeling basis and also some modeling tools that will be available in ModelCiências, which is a portal for the project Semiquantitative and Quantitative Computational Modeling in Science Education of CNPq's Research and Post-graduation Southern Plan. The article presents, also, the characteristics of the courses that will be avaliable in the portal, technical aspects of its construction and the current stage of development of the project.

Key-words: Modeling, computer, computational tools.
\end{abstract}

\title{
Introdução
}

O ModelCiências é o portal do projeto Modelagem Computacional Semiquantitativa e Quantitativa na Educação em Ciências do Plano Sul de Pesquisa e Pós-graduação do CNPq - PSPPG. Este empreendimento, financiado pelo $\mathrm{CNPq}$, consiste na implantação da formação de professores à distância, tendo como referencial o Aprendizado Exploratório de Ciências através da modelagem computacional. Pretendemos oferecer ao professor do ensino fundamental e médio um caminho seguro para a introdução da Informática Educativa na escola. De nada adianta políticas governamentais com a finalidade de introduzir o computador no ambiente escolar, de cima para baixo, se o professor não estiver preparado para esse desafio, com uma filosofia clara de trabalho e um conjunto de ferramentas computacionais de fácil domínio e acesso.

São parceiros no desenvolvimento do trabalho os professores Arion de Castro Kurtz dos Santos (dfsarion@furg.br), da Fundação Universidade Federal do Rio Grande (FURG), coordenador geral, com seu grupo de pesquisa, e os coordenadores locais, com seus grupos de pesquisa: Laércio Ferracioli (laercio@npd.ufes.br), da Universidade Federal do Espírito Santo (UFES); Fábio Ferrentini Sampaio (ffs@ @nce.ufrj.br), da Universidade Federal do Rio de Janeiro (UFRJ); José Francisco Dias da Fonseca (jfrandef@ufpel.tche.br), da Universidade Federal de Pelotas (UFPEL). A filosofia de construção deste portal tem como base FERRACIOLI (2001). 


\section{A dinâmica geral da proposta}

Nossa proposta possui as dimensões de pesquisa e de aperfeiçoamento de professores através da modelagem computacional. Com os recursos financeiros do $\mathrm{CNPq}$ adquirimos um servidor profissional COMPAQ, onde está localizado este portal. Ainda deveremos adquirir microcomputadores que deverão ser distribuídos entre as instituições da Rede, em nosso caso particular FURG e UFPEL. Através da interação entre os demais pesquisadores da Rede deveremos desenvolver e aperfeiçoar os cursos virtuais, e com a interação pesquisadores-professores da rede de ensino, em cursos de aperfeiçoamento virtuais e presenciais, que agora estaremos oferecendo a comunidade, pretendemos disponibilizar os materiais desenvolvidos. O resultado da interação com os professores da rede de ensino permitirá aos pesquisadores participantes da Rede a realização de pesquisas sobre o Aprendizado Exploratório de Ciências.

\section{Aspectos técnicos da construção do ModelCiências}

FIGURA 1- TELA COM A ENTRADA DO PORTAL DO MODELCIÊNCIAS (VERSÃO EXPERIMENTAL)

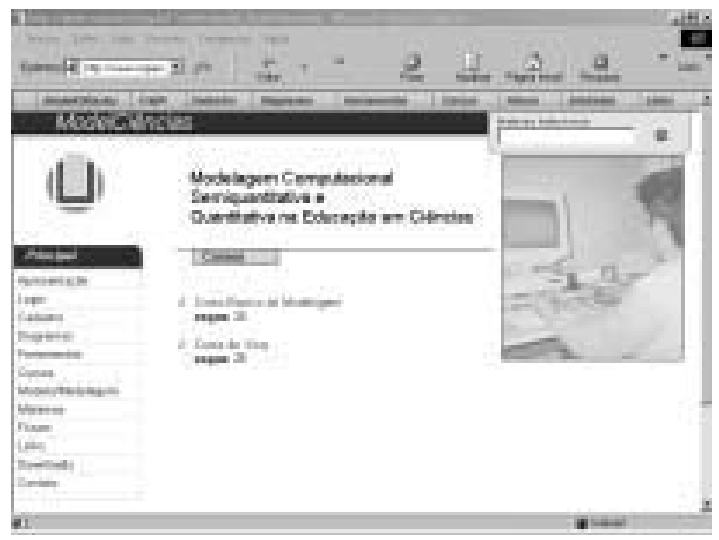


O ModelCiências (figura 1) é um sistema de ensino à distância, que tem como filosofia a modelagem através da Dinâmica de Sistemas, com o intuito de fazer com que professores utilizem os cursos disponíveis para auxiliar na implementação da informática educativa nas escolas. Cursos com esta proposta já foram desenvolvidos de modo presencial na FURG em uma série de oportunidades, e tinham sempre a necessidade de se alocar recursos como salas de aula, material didático e professores, além de limitar o número de participantes por diversos fatores. O ModelCiências deverá disponibilizar estes cursos através da web, tornando mais fácil e flexível a participação de professores, já que os horários são estabelecidos pelos próprios usuários do sistema, podendo também beneficiar pessoas de diferentes localidades. A implementação do sistema mostrou-se viável devido à utilização de software livre na construção das ferramentas e do site. Para o desenvolvimento do sistema estão disponíveis o servidor COMPAQ, executando o sistema operacional Linux, e um laboratório para o desenvolvimento do material instrucional, dos cursos on-line e do próprio site do ModelCiências. A política de construção do site é baseada em linguagens de programação como Php e Java, e o banco de dados utilizado é o MySQL. Estas ferramentas mostram uma boa integração e se adaptam perfeitamente às necessidades do sistema. O Php oferece a possibilidade de construção de páginas HTML dinâmicas, tornando possível a interatividade existente nos cursos. O Java está sendo utilizado para construção de softwares de modelagem, além de contribuir para o site com Applets e JavaScripts. O MySQL é o responsável pelos dados armazenados no sistema, sendo que a boa integração com o Php e Java também favorece a utilização deste banco de dados. No site do ModelCiências, o usuário deve cadastrar-se para que possa utilizar os recursos disponíveis. O cadastro tem como objetivo, além de manter um controle de acessos aos cursos, coletar as informações necessárias para que os tópicos mais diretamente ligados à área de atuação do usuário sejam sugeridos ao mesmo. Os cursos são divididos em módulos que contém uma apresentação teórica referente aos mesmos, além de questões a serem resolvidas. Questões subjetivas também estão presentes no site e, para que estas possam ser discutidas, há um fórum que é dividido por assunto, além de ter várias seções específicas para alguns módulos dos cursos. O portal ModelCiências, embora disponibilize cursos sobre modelagem, não é um curso específico, mas consiste em um editor de cursos à distância, onde o professor autorizado pode criar e disponibilizar seus próprios cursos. Os alunos matriculados também acessam estes cursos atra- 
vés do portal. Para a criação dos cursos o ModelCiências disponibiliza os seguintes componentes:

a) biblioteca de materiais instrucionais - um repositório para qualquer tipo de arquivo (arquivos texto, pdf, figuras, animações, modelos etc) que são colocados pelo professor do curso e são disponibilizados para os alunos;

b) fórum - algumas salas para discussão (figura 2) de tópicos relacionados ao curso, exclusivos do ModelCiências, e de assuntos genéricos;

FIGURA 2 - TELA COM PARTE DO FÓRUM DO MODELCIÊNCIAS (VERSÃO EXPERIMENTAL)

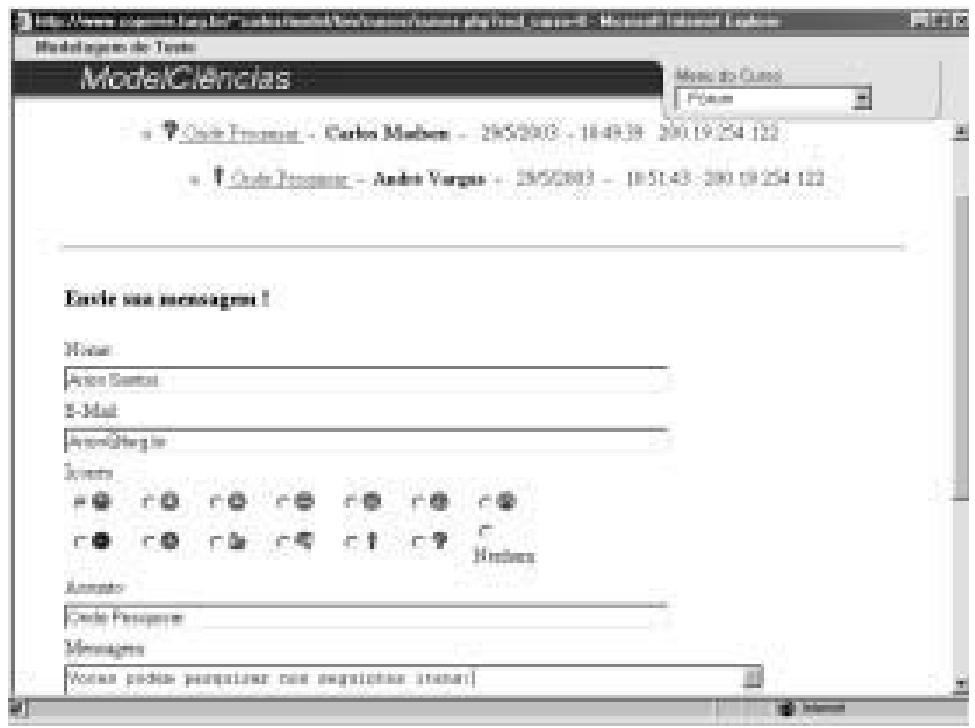

c) chat - existem várias salas, semelhantes ao Fórum, para discussão em tempo real;

d) módulo para criação de avaliações - através deste componente (figura 3), o professor pode criar avaliações bastante flexíveis e disponibilizálas para os alunos. As respostas para as questões podem ser em formato texto ou um arquivo para o caso de figuras e modelos. O professor escolhe o peso de cada questão, a data de entrega e formula um enunciado em formato HTML. Após as questões serem respondidas, o professor tem acesso a elas para que sejam corrigidas; 
FIGURA 3 - TELA COM A AVALIAÇÃO NO MODELCIÊNCIAS (VERSÃO EXPERIMENTAL)

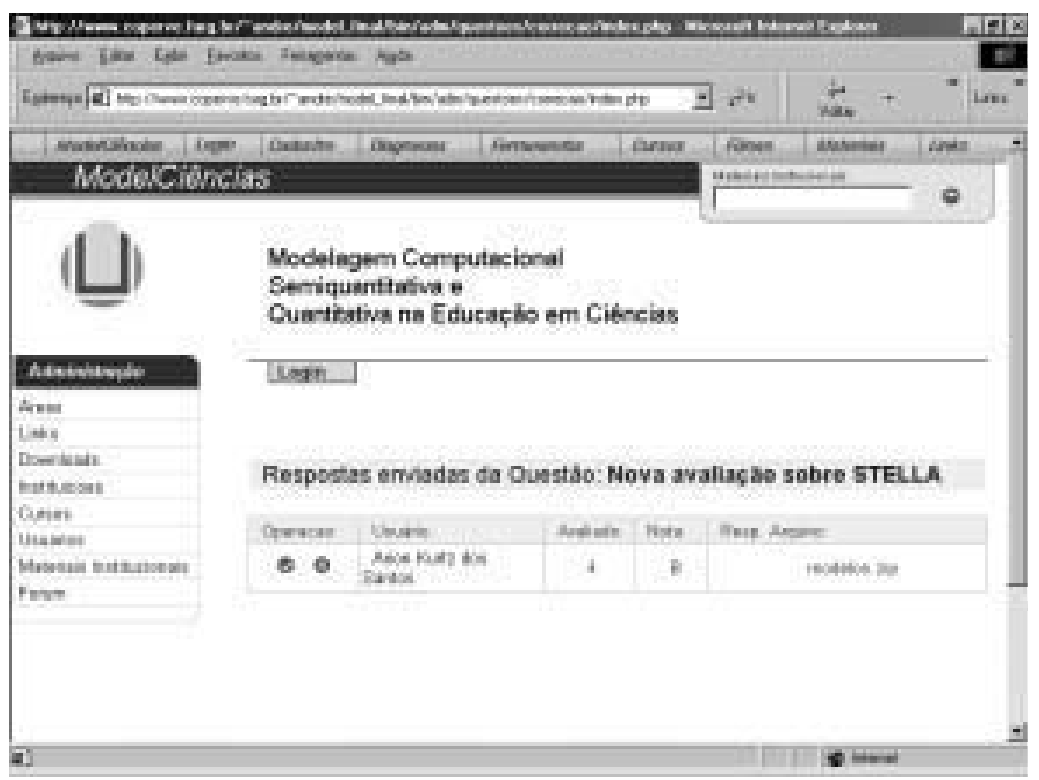

e) setor para tirar dúvidas - o aluno tem disponível um componente onde pode enviar dúvidas sobre o curso para o professor e este pode responde-las. O aluno escolhe se as dúvidas podem ou não ser visíveis a outros colegas do curso;

f) mural para o professor - o professor tem a sua disposição um mural onde coloca os avisos de novos materiais instrucionais, novas avaliações, marca encontros de chat etc. As entradas do mural são automaticamente enviadas por e-mail a todos os alunos do curso. 


\section{Fundamentação teórica do projeto}

Desde 1992 nosso grupo tem se dedicado, dentro do projeto Profecomp, ${ }^{2}$ ao trabalho com a Dinâmica de Sistemas, tendo como base o desafio de levar, aos alunos do ensino fundamental, médio e superior, os princípios dessa área. Nossa preocupação tem sido predominantemente educativa.

Nossa fundamentação teórica tem sido baseada nos Fundamentos Psicológicos da Modelagem (VYGOTSKY, 1993), na Modelagem Semiquantitativa, com Visq (SANTOS, 1995) e Wlinkit (SAMPAIO, 2000), na Modelagem Quantitativa, com Stella (RICHMOND et al., 1987) e na Modelagem em nível de Objetos, com Autocel-RCO (ARAUJO, 2000) e World Maker (BOOHAN, 1994). Entendemos a modelagem como uma área com potencial para o trabalho conforme os saberes propostos por MORIN (2000). Nossos cursos à distância deverão seguir alguns dos princípios discutidos por BELLONI (2001) e FERRACIOLI (2001).

\section{Modelo e modelagem}

Para entender a natureza, o homem, desde os tempos mais remotos, tem procurado por regularidades e tem desenvolvido modelos, que podem ser entendidos como a codificação dessas regularidades.

Um modelo cristaliza, momentaneamente, as relações causais através de uma topologia específica, passando a existir como um mundo artificial (MELLAR et al., 1994). Esse mundo, então, transforma-se num objeto de análise que estará disponível para exploração, e mesmo modificação pelos estudantes. A modelagem, dentro dessa perspectiva, aparece como mais um possível componente do processo de ensino e aprendizagem.

Modelagem é o processo de estabelecer relações entre entidades importantes de um sistema. Para cada modelador existe um modelo mental básico (a visão ou imagem que o modelador tem do sistema real ou virtual)

2 Projeto Desenvolvimento e Uso de Ferramentas Computacionais para o Aprendizado Exploratório de Ciências - http://www.sf.dfis.furg.br/profecomp 
a partir do qual é construído um modelo específico simplificado. Ao experimentar esse modelo simplificado, desejamos aumentar o entendimento do modelo base e também do sistema real ou virtual caracterizado por esse modelo. Um modelo deve ser construído selecionando-se o menor subconjunto de variáveis que descrevem adequadamente o sistema real ou virtual. A habilidade em modelagem depende de experiência, conhecimento, intuição, julgamento, percepção e imaginação. A construção de um modelo é baseada num modo específico de olhar para o objeto, isto é, para a "realidade".

Existem dois modos distintos, mas complementares, para utilizar uma ferramenta computacional de modelagem - o exploratório e o expressivo. No modo exploratório, o estudante explora um modelo já colocado no computador, como uma simulação. Nesse caso, ele explora as representações, desenvolvidas pelos professores ou pesquisadores, que podem ser distintas de suas próprias representações. No modo expressivo, o estudante desenvolve seus modelos, apresentando suas próprias representações da "realidade" que está sendo modelada.

Ao apresentarmos o modelo para ser explorado (atividade exploratória), como uma simulação, desenvolveremos um conjunto de atividades onde constem um certo número de questões do tipo: $O$ que acontece se ...? e Por que você pensa que é isso que acontece? Questões do tipo $O$ que acontece se...? visam explorar o raciocínio suposicional, fundamental no processo de aprendizagem por tentativa e erro, e da descoberta pela tentativa de alternativas. Questões do tipo Por que você pensa que é isso que acontece? visam buscar explicações causais sobre como o estudante pensa a respeito do modelo e da "realidade" que está sendo descrita (SANTOS, 1995).

Ao desenvolver um modelo, o aluno, a partir das interações sociais, internaliza suas ações e constrói modelos mentais sobre algum fenômeno do mundo real ou virtual. Esse processo pode ser lento, e os modelos encontram-se dentro de sua mente. É solicitado que, utilizando uma ferramenta de modelagem (hardware e software específico), este aluno dê início a um processo iterativo de externalização do conteúdo dos modelos mentais. Um modelo computacional inicial é criado e, agora, ele pode pensar sobre seu pensamento. Ao fazer isto, ele começa a internalizar o comportamento descrito no modelo, iniciando um processo de comparação desse comportamento com o esperado pelos modelos mentais.

Enquanto o aluno não estiver satisfeito com o comportamento internalizado pela ação sobre o modelo computacional, ele dá início a uma 
nova externalização com o intuito de aperfeiçoar o modelo computacional. Ao fazer isto, ele, ao ser "perturbado" pelo professor ou pesquisador, conscientiza-se sobre a realidade e o modelo e passa a entendê-los melhor. O processo se repete até que o comportamento do modelo de simulação seja uma aproximação razoável e satisfatória do comportamento do fenômeno do mundo real ou virtual, conforme internalizado pelo aluno. Na comparação entre o mundo real ou virtual e o modelo, criado pelo aluno, é que os pesquisadores começam a entender mais o conteúdo dos modelos mentais do aluno (idéias baseadas em VYGOTSKY, 1993).

Entendemos a modelagem como uma área que permite o trabalho com o global, o multidimensional e o complexo, conforme os saberes necessários à educação do futuro propostos por MORIN (2000). Nossos cursos à distância do portal do ModelCiências serão complementados com encontros presenciais, centrados no aluno, com ênfase nas tecnologias interativas, e com a disponibilização de monitores, seguindo os demais princípios discutidos por BELLONI (2001). Do ponto de vista do aprendizado exploratório de Ciências, seguiremos FERRACIOLI (2001) que considera como os principais componentes para a Educação a Distância a tecnologia da informação, o conteúdo curricular específico de Ciências (Física, Química, Biologia e Ambientais) e a modelagem como uma estratégia para elicitação e construção do conhecimento através da utilização de ambientes de modelagem computacional.

\section{Diagramas Causais ou de Influências como os primitivos da mo- delagem computacional}

Diagramas Causais são também chamados de Diagramas de Influências. Estes diagramas podem ser considerados os primitivos das ferramentas computacionais que serão disponibilizadas no ModelCiências. Há pouca ou nenhuma diferença, mas os Diagramas Causais podem ser pensados como Diagramas de Influências que não mostram detalhes maiores. Os Diagramas de Influência (veja um exemplo na figura 4) podem ser pensados como Diagramas Causais detalhados (COYLE, 1988). 
FIGURA 4 - DIAGRAMA DE INFLUENCIA PARA O FUNCIONAMENTO DE UM AQUECIMENTO CENTRAL*

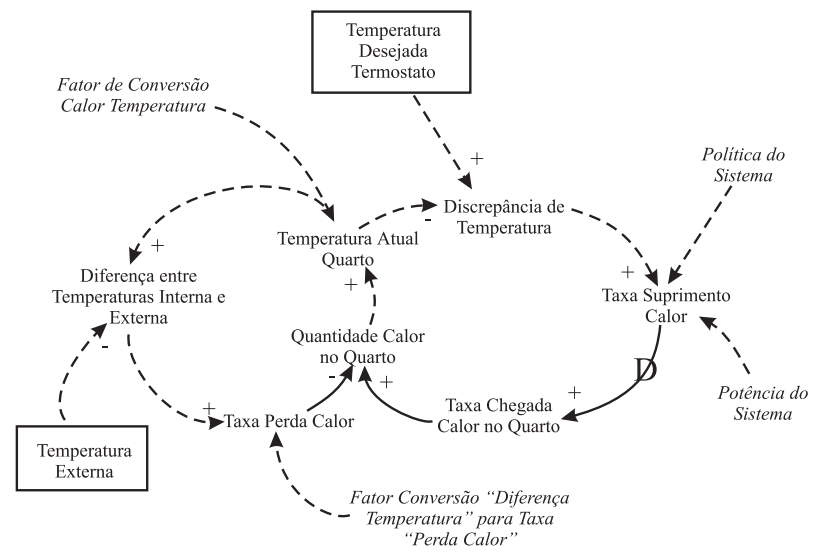

NOTA: *Sobre o entendimento dos elos positivos e negativos veja SANTOS (1995).

Os modelos mentais dos sistemas dinâmicos são mal definidos, não são fáceis de comunicar, e a natureza imprecisa da linguagem pode ser usada para esconder uma imagem mental nebulosa do falante e do ouvinte. Os modelos mentais não podem ser manipulados efetivamente, pois não é possível "manejar" na mente, imediatamente, todas as facetas de um sistema complexo. Temos a tendência de fragmentar o sistema em pedaços e tirar conclusões separadamente dos subsistemas, e tais fragmentações não mostram como os subsistemas interagem.

Quando um sistema é reduzido a um diagrama de elos causais e equações matemáticas, ele pode, então, ser examinado e comunicado a outros, sendo, assim, possível computar a evolução temporal de variáveis, de tal modo que podemos tentar entender melhor a "realidade".

A construção de diagramas de elos causais permite a uma pessoa comunicar, com poucas palavras e setas, a sua teoria da estrutura subjacente ao problema. A técnica também ajuda o estudante a expressar seu nível de entendimento de uma situação, algumas vezes referido como seu modelo mental. O raciocínio causal é a chave para organizar idéias num estudo de Dinâmica de Sistemas (FORRESTER, 1973; 1990³). 


\section{Ferramentas Computacionais do ModelCiências}

\section{Ferramentas Computacionais Icônicas > Quantitativas}

Modelos quantitativos são utilizados em processos de decisão, em gerenciamento, não com o objetivo de fazermos previsões, mas de estudarmos possíveis cenários. A modelagem quantitativa é a representação do raciocínio quantitativo através de ferramentas computacionais icônicas como Stella (RICHMOND et al., 1987) ou não icônicas como, por exemplo, o IAT (KLEER et al., 1997; MARTINS, 2002) que forneçam resultados numéricos. Quando utilizamos esse tipo de modelagem estamos interessados em saber exatamente de quanto foi uma certa variação. A modelagem quantitativa tem sido mais utilizada em áreas como a Física, por exemplo.

\section{Ferramentas Computacionais Icônicas > Quantitativas > Stella}

Stella, acrônimo para Structural Thinking Experimental Learning Laboratory with Animation ${ }^{4}$ (RICHMOND et al., 1987) é uma ferramenta de modelagem quantitativa que utiliza a metáfora de tanques, válvulas e canos. Atualmente, está disponível a última versão para Windows, demonstrativa (no save), no seguinte endereço:

http://www.hps-inc.com/education/new-stella.htm

FIGURA 5 - DIAGRAMA NO STELLA. UM TANQUE X COM UMA TAXA DE SAÍDA DXDT (REPRESENTANDO DX/DT). TAXA DE VARIAÇÃO TEMPORAL DE $X$

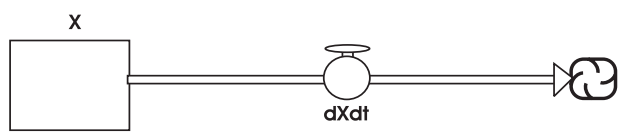

No Stella, um tanque $\square$ (estoque ou nível) representa uma quantidade cujo valor inicial pode crescer ou decrescer. É conveniente descrever

4 Tradução adaptada: Laboratório de Aprendizagem Experimental com Animação para o Pensamento Sistêmico. 
as variáveis relacionadas a acumulações durante a passagem do tempo por tanques (ou estoques). Uma torneira $\bigcirc$ (taxa) conectada a um tanque decide quão rápido a quantidade no tanque está mudando. Várias torneiras podem ser conectadas a um tanque. Quantidades representadas por um círculo (conversores) podem ser constantes, ou podem ser calculadas de outras quantidades.

Stella permite a construção de um modelo através da conexão desses objetos básicos e o usuário não necessita pensar sobre que linhas de programa escrever. Ele tem que fornecer relações algébricas, mas o sistema converte essas relações em linhas de programa. Stella permite a obtenção de gráficos de quaisquer variáveis contra outras, e contra o tempo, e gera uma tabela de dados.

\section{Ferramentas Computacionais > Semiquantitativas}

Ao tentarmos responder questões envolvendo Física ou outra ciência, freqüientemente desenvolvemos raciocínios não numéricos, do tipo: se a massa aumenta então a força aumenta... ou se a distância diminui então a força aumenta..., ou ainda, se eu empurro o carrinho ele deslizará sobre a mesa... São raciocínios em que esperamos uma modificação em termos de aumento ou diminuição do valor de uma entidade ou variável, embora não estejamos ainda preocupados em saber exatamente de quanto será essa variação. Esse tipo de raciocínio, sem precisão, em que valores numéricos não estão envolvidos, costuma ser chamado de raciocínio semiquantitativo.

A modelagem semiquantitativa é a representação do raciocínio semiquantitativo através de diagramas causais ou de influência - com lápis e papel - ou ferramentas computacionais que permitem o desenvolvimento desses diagramas - Visq e Wlinkit - e dos diagramas de fluxo - com taxas e níveis - (mapeamento Stella) na tela do computador. Não há resultados numéricos, apenas mudanças nos estados semiquantitativos das entidades representadas.

\section{Ferramentas Computacionais $>$ Semiquantitativas $>$ Visq}

Variáveis que Interagem de Modo Semiquantitativo - Visq - utiliza a matemática das redes neurais para animar diagramas causais na tela do computador. Visq fornece uma interpretação sistemática a qualquer diagra- 
ma causal, permitindo a criação de modelos semiquantitativos sem levar em consideração o conteúdo, tanto nas ciências naturais como humanas.

A fundamentação das redes neurais do Visq trabalha considerando que cada par X à $\mathrm{Y}$ (X afeta positivamente $\mathrm{Y}$, veja a figura 6) representa que o estado semiquantitativo, ou nível vertical de $\mathrm{X}$, causa uma mudança no nível vertical de Y. Quer dizer, X é a taxa de variação temporal de Y.

FIGURA 6 - PAR X AFETA POSITIVAMENTE Y EM VISQ COM A CORRESPONDENTE SAÍDA GRÁFICA DE Y VERSUS TEMPO*

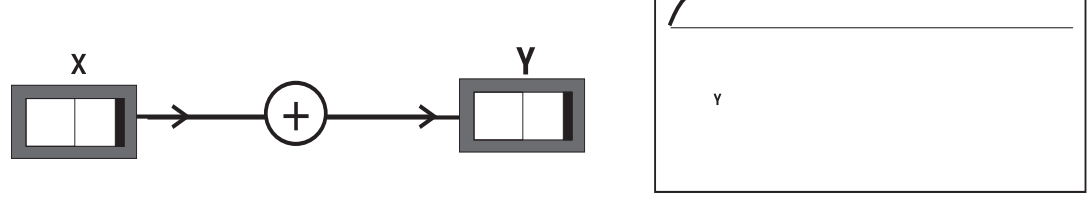

NOTA: *Note que Y aumenta, mas acaba amortecendo. A linha horizontal corresponde ao nível normal (igual a zero) e coincide com o eixo dos tempos.

Resumidamente, um par positivo, como o apresentado na figura 6, seria descrito por uma equação semelhante a

$$
\frac{d Y}{d t}=k^{*}\left(1-Y^{2}\right) * x-\left(p^{*} Y\right)
$$

onde $\mathrm{k}$ e p são constantes, limitando os níveis das variáveis envolvidas a uma variação entre -1 e +1 . Veja que o nível de $\mathrm{Y}$ acabará amortecendo com o passar do tempo. Assim, as redes ou diagramas causais, sempre evoluirão para um estado estacionário, de equilíbrio. Uma pessoa, analisando um diagrama causal feito com lápis e papel, poderá prever diversos comportamentos dinâmicos como possibilidades viáveis de solução. Em Visq, um diagrama causal terá uma interpretação única.

Evidentemente, um modelo em Visq poderá conter muitas caixas representando variáveis com elos negativos e positivos entre elas. A matemática fica escondida do usuário. 
FIGURA 7 - TELA MOSTRANDO A VERSÃO EXPERIMENTAL DO NOVO VISQ

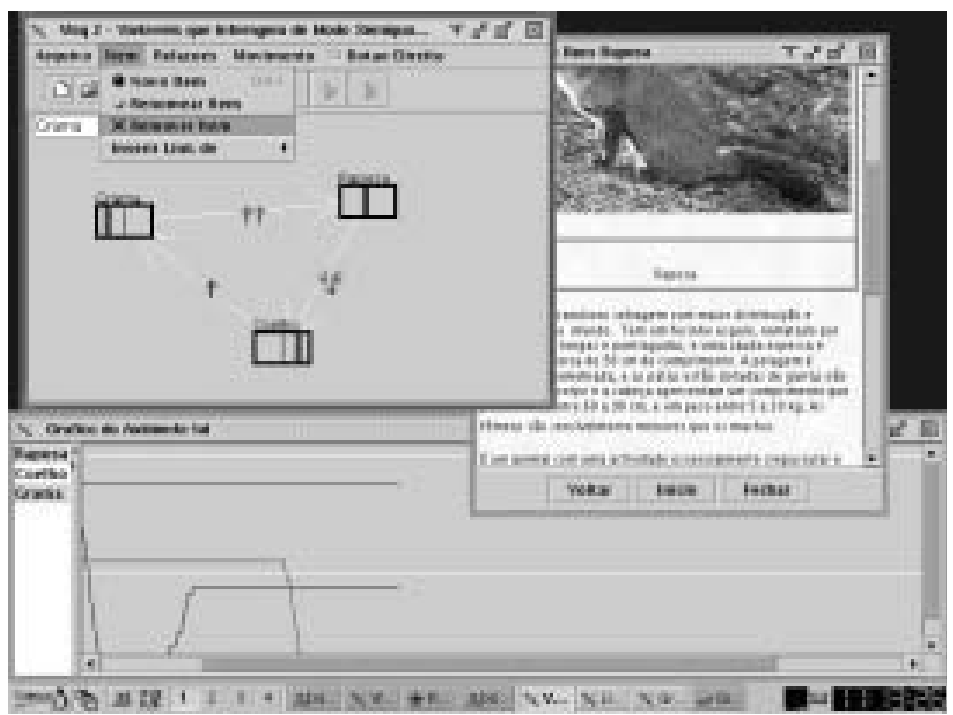

O novo Visq (figura 7) está intimamente ligado ao portal ModelCiências. Através do Visq o aluno é capaz de enviar seus modelos que serão avaliados pelo professor. Esta ferramenta possui muitos recursos novos, entre eles destacamos a possibilidade de, através das entidades (caixas no Visq), realizar um trabalho com hipertextos que correspondem a documentos HTML, podendo estes estar na máquina do usuário ou em qualquer outro servidor conectado à rede, inclusive serem materiais instrucionais do Portal. O novo Visq foi desenvolvido em Java, o que garante a sua portabilidade, permitindo que qualquer aluno possa usá-lo independentemente de seu sistema operacional. Visq utiliza os recursos de Threads, o que nos permite a visualização de gráficos simultâneos e a alteração do modelo e dos gráficos enquanto o modelo está sendo animado. Todas estas características representam um grande avanço com relação ao Visq antigo e são trabalho direto dos bolsistas do ModelCiências. Contudo, salientamos que ainda é possível para o usuário enviar, se necessário, modelos desenvolvidos no antigo Visq para o servidor. 


\section{Características gerais dos cursos disponíveis no ModelCiências}

Os cursos disponíveis no ModelCiências contêm um módulo básico, totalmente interativo, sobre os fundamentos da Dinâmica de Sistemas, obrigatório para os professores de todas as áreas, uma vez que apresenta a linguagem dos diagramas causais e de fluxo, essenciais para o entendimento das ferramentas computacionais e materiais instrucionais disponíveis. Após a conclusão do módulo básico, o professor será dirigido para o conjunto de cursos compatíveis com a atuação profissional ou escolha. Poderá cursar módulos de áreas distintas à formação básica, se for de seu interesse, mas deverá concluir um conjunto de cursos de cada vez. Os módulos específicos foram classificados como pertencentes às áreas de Ciências Exatas, Biológicas e Humanas. A escolha deverá ser feita no momento do cadastro on-line no curso. O ModelCiências contém bancos de dados com as ferramentas computacionais (softwares), os materiais instrucionais em PDF/HTML, livros, teses e dissertações sobre modelagem. Dentro de cada módulo há informações sobre como o professor deverá proceder para instalar os principais softwares e materiais instrucionais no computador pessoal. Há informações de como proceder para evoluir no entendimento de algum aspecto particular da modelagem e links para o aprofundamento do domínio dessa estratégia de ensino. As atividades propostas no material instrucional têm como meta fornecer um conjunto de técnicas que certamente ajudarão no futuro, quando da utilização da modelagem com seus próprios alunos. O portal do ModelCiências apresenta diversos links interessantes e úteis para a formação do professor.

\section{Cursos > Curso base}

Todos os professores inscritos deverão realizar o módulo básico, obrigatório, sobre os fundamentos da Dinâmica de Sistemas, uma vez que este módulo inicial apresenta a linguagem dos diagramas causais e de fluxo, essenciais para o entendimento das ferramentas computacionais e materiais instrucionais disponíveis. O módulo básico, interativo, contém Applets em Java para o desenvolvimento das atividades. Os módulos dos cursos específicos serão compostos por atividades exploratórias e expressivas. 


\section{Cursos > Ciências Exatas}

No curso da área de Ciências Exatas, o professor trabalhará com atividades sobre diagramas de influência para o sistema de aquecimento central (figura 4), inicialmente com lápis e papel, e posteriormente através da modelagem semiquantitativa das situações com o programa Visq. Desenvolverá modelos físicos com o Wlinkit (SAMPAIO, 2000) como, por exemplo, o funcionamento de um termostato e outros modelos que envolvam variáveis do tipo gatilho. Desenvolverá o estudo do mapeamento semiquantitativo em termos de taxas e níveis e de modelos quantitativos com o programa Stella para situações como, por exemplo, em Física, os movimentos, o oscilador harmônico, as equações de Lorenz para a turbulência na atmosfera e o Caos determinístico. O professor será encorajado a desenvolver modelos quantitativos, em Física, com softwares históricos como o CMS (HOLLAND, 1988) e as Planilhas Eletrônicas. Terá, também, a oportunidade de trabalhar com propostas específicas de utilização de softwares quantitativos sem representação icônica como o IAT, para a Investigação dos Acidentes de Trânsito, que se constitui em uma proposta interessante para o ensino médio de Física. Para melhorar a formação, terá a oportunidade de aprofundar os estudos com textos específicos de livros sobre aplicações do Stella em Física, com a leitura de dissertações que envolvam a utilização deste programa e com a visita a links relevantes.

\section{Cursos > Ciências Humanas}

No curso da área de Ciências Humanas, o professor desenvolverá modelos qualitativos, tendo como base a representação por hexágonos (HODGSON, 1994) para situações socioambientais apresentadas através de textos e explorará modelos socioambientais de outros autores e desenvolverá seus próprios modelos com Visq. Desenvolverá modelos sociais com o Wlinkit como, por exemplo, a descrição da dinâmica de uma revolução ou o entendimento de textos sobre a teoria de Freud e outros modelos que envolvam variáveis do tipo gatilho. Desenvolverá, também, o estudo do mapeamento semiquantitativo em termos de taxas e níveis com o programa Stella em diversas situações como, por exemplo, a dinâmica do stress, e modelos quantitativos com este programa para situações como, por exemplo, em Gerenciamento, empresas que contratam vendedores em função das encomendas dos clientes. Para melhorar a formação, terá a oportunidade de aprofundar os estudos com a leitura de textos específicos de artigos 
e dissertações sobre aplicações socioambientais de Visq e com a visita a links relevantes.

\section{Cursos > Ciências Biológicas}

No curso da área de Ciências Biológicas, o professor desenvolverá modelos Ecológicos com o Visq, como os da pesca do camarão e da poluição em Rio Grande (cidade litorânea do Rio Grande do Sul), e com o Wlinkit como, por exemplo, o funcionamento da pupila do olho e outros modelos que envolvam variáveis do tipo gatilho. Desenvolverá o estudo do mapeamento semiquantitativo em termos de taxas e níveis com o programa Stella e modelos quantitativos com este programa em diversas situações como, por exemplo, em Ecologia, a interação presa-predador e outros modelos clássicos apresentados na obra de ODUM (1985). Desenvolverá modelos sobre interação entre espécies com o Autômato Celular AutcelRCO (ARAUJO, 2000) e outros autômatos disponíveis como o World Maker (BOOHAN, 1994). Para melhorar a formação, terá a oportunidade de aprofundar os estudos com textos específicos de livros sobre aplicações ecológicas do Stella, com a leitura de dissertações que envolvam a utilização do programa Visq e com a visita a links relevantes.

\section{Conclusão}

No atual momento (junho de 2003) temos dois bolsistas de Apoio Técnico do CNPq e um bolsista voluntário, todos do curso de Engenharia da Computação, trabalhando no aperfeiçoamento do curso base, do portal editor de cursos à distância e da nova versão do Visq apresentada. Temos também um bolsista de Iniciação Científica do CNPq trabalhando no desenvolvimento de alguns dos cursos específicos mencionados. Em breve esperamos estar em condições de divulgar o curso a distância para os professores da comunidade. Dependendo dos recursos a serem liberados pelo $\mathrm{CNPq}$, daremos início a formação de turmas específicas de professores que serão atendidos através de um curso de extensão já registrado oficialmente na FURG. 
Esperamos, a partir do material proveniente da interação com os professores da rede de ensino, obter dados que permitam a realização de pesquisas sobre o Aprendizado Exploratório de Ciências.

\section{REFERÊNCIAS}

ARAUJO, I. S. Autcel-RCO - uma alternativa para o raciocínio com objetos. Revista Eletrônica Mestr. Educ. Ambient., Rio Grande, 2000. Disponível em: <http:// www.sf.dfis.furg.br/mea/remea/anais3/artigo3.htm> v. especial - Versão Eletrônica dos Anais do "III Seminário Sobre Representações e Modelagem no Processo de EnsinoAprendizagem", 2000.

BELLONI, M. L. Educação a distância. Campinas: Autores Associados, 2001. (Coleção Educação Contemporânea).

BOOHAN, R. Creating Worlds from Objects and Events. In: MELLAR, H. et al. (Eds.). Learning with Artificial Worlds: Computer Based Modelling in the Curriculum. London: The Falmer Press, 1994.

CAMILETTI, G. G.; FERRACIOLI, L. A Utilização da modelagem computacional quantitativa no ensino de Física. Rev. Eletrônica Mestr. Educ. Ambient., Rio Grande, 2000. Disponível em: 〈http://www.sf.dfis.furg.br/mea/remea/anais3/artigo6.htm〉

COYLE, R. G. System Dynamics Modelling. A practical approach. London: Chapman \& Hall, 1988.

FERRACIOLI, L. Aprendizagem exploratória em ciência através da educação a distância. Vitória: Laboratório de Tecnologias Interativas Aplicadas à Modelagem Cognitiva, Departamento de Física, Universidade Federal do Espírito Santo, 2001.

FORRESTER, J. W. World Dynamics. Cambridge, Mass.: Wright-Allen Press Inc., 1973. . Principles of systems. Cambridge: Wright-Allen Press Inc., 1971.

. Principles of Systems. Portland, OR: Productivity Press, 1990.

HODGSON, A. M. Hexagons for Systems Thinking. In: MORECROFT, J. D. W.; STERMAN, J. S. (Eds.). Modelling for Learning Organizations. Portland, OR: Productivity Press, 1994.

HOLLAND, D. A software laboratory. Dynamical Modelling and the Cellular Modelling System. School Science Review, p. 406-416, mar. 1988. 
KLEER, A. A.; THIELO, M. R.; SANTOS, A. C. K. dos. IAT - um software dedicado à investigação de acidentes de trânsito. Caderno Catarinense de Ensino de Física, v. 14, n. 2, p. 160-169, ago. 1997.

MARTINS, M. Z. IAT - Investigando acidentes de trânsito - desenvolvimento de um software para o ensino de Física em possível aplicação a situações reais de acidentes. In CONGRESSO DE INICIAÇÃO CIENTÍFICA DA FURG, 10., 2002, Rio Grande. Resumos... Rio Grande: Ed. da FURG, 2002. p. 23.

MELLAR, H. et al. (Eds.). Learning with Artificial Worlds: Computer Based Modelling in the Curriculum. London: The Falmer Press, 1994.

MORIN, E. Os sete saberes necessários à Educação do futuro. São Paulo: Cortez, 2000.

ODUM, E. P. Ecologia. Rio de Janeiro: Ed. Guanabara, 1985.

OGBORN, J.; WONG, D. A Microcomputer Dynamical Modelling System. Physics Education, v. 19, p. 138-142, 1984.

RICHMOND, B. et al. An Academic User's Guide to Stella. Lyme: High Performance System Inc., 1987.

SAMPAIO, F. F. Modelagem dinâmica computacional e o processo de ensino-aprendizagem: algumas questões para reflexão. Revista Eletrônica Mestr. Educ. Ambient., Rio Grande, Volume Especial - Versão Eletrônica dos Anais do "III Seminário Sobre Representações e Modelagem no Processo de Ensino-Aprendizagem, 2000”. Disponível em: <http://www.sf.dfis.furg.br/mea/remea/anais3/artigo2.htm>

SANTOS, A. C. K. dos. Introdução à modelagem computacional na Educação. Rio Grande: Ed. da FURG, 1995.

VYGOTSKY, L. S. Pensamento e linguagem. São Paulo: Martins Fontes, 1993.

Texto recebido em 25 maio 2003

Texto aprovado em 05 set. 2003 